\title{
Correlation of Erythrocyte Distribution Width Values with Preeclampsia and Preeclampsia with Severity Criteria
}

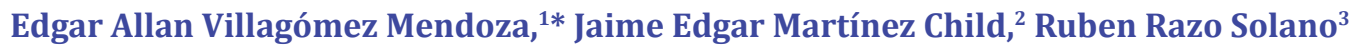 \\ ${ }^{1}$ Fourth year resident physician of Gynecology and Obstetrics \\ ${ }^{2}$ Fourth year resident physician of Gynecology and Obstetrics \\ ${ }^{3}$ Obstetrician Gynecologist
}

\begin{abstract}
Aim: To determine the correlation of the red cell distribution width values with pre-eclampsia and pre-eclampsia with severity criteria.

Materials and methods: Cross-sectional, prospective study of patients who met the inclusion criteria in the period from August 2019 to August 2020. Statistical analysis was performed using Student's t test and Spearman's correlation to calculate the dependence between the non-parametric variables, the value of $\mathrm{p}<0.05$ was considered statistically significant.
\end{abstract}

Results: 9 patients with pre-eclampsia and 30 pre-eclampsia were obtained with severity criteria. There was no statistical difference when comparing the RDW of patients with pre-eclampsia and pre-eclampsia with severity criteria $(\mathrm{p}=0.226)$, in patients with systolic blood pressure values $\geq 160 \mathrm{mmHg}$ there was an increase in RDW levels ( $\mathrm{p}=0.042$ )

Objective: To determine the correlation of erythrocyte distribution width values with pre-eclampsia and pre-eclampsia with severity criteria.

Conclusions: There is a significant relationship between increased RDW and systolic blood pressure values $\geq 160 \mathrm{mmHg}$.

Keywords: Preeclampsia, Preeclampsia with severity criteria, Red blood cell distribution width, Hypertensive states of pregnancy

\section{Background}

Preeclampsia is a multisystemic disorder characterized by hypertension and proteinuria, which occur for the first time after 20 weeks of gestation, it is one of the main causes of maternal morbidity and mortality and perinatal worldwide. ${ }^{1}$ Hypertensive disorders of pregnancy affect approximately $15 \%$ of pregnancies and represent $18 \%$ of maternal deaths in the world, in Mexico according to the Ministry of Health, the Preeclampsia accounts for up to $34 \%$ of all maternal deaths. ${ }^{2}$ Although it is characterized by alteration in the remodeling of the spiral arteries whose origin is an alter- ation in the invasion of the extravillous trophoblast, conditioning placental hypoperfusion that generates reactive oxygen species and cytokines pro-inflammatory, ${ }^{3}$ in such a way that chronic hypoperfusion of the maternal-fetal circulation could compromise the erythrocytes, this whose main function is the transport of oxygen, for which the erythrocyte distribution width (RDW) describes the percentage variation in the size of the erythrocytes, Values between $11 \%$ and $15 \%$ are generally considered normal. A value above this range indicates a heterogeneous population of erythrocytes, that is, increased RDW values are related to when there is excessive destruction of red blood cells or a deficit in its production. ${ }^{4}$

\begin{tabular}{|l|l|}
\hline \hline Quick Response Code: & Forresponding author: Edgar Allan Villagómez Mendoza, Lake Nyasa 46 Col. Jardines de \\
Morelos, Ecatepec, State of Mexico, CP 55070 \\
Received: 22 November, 2021 \\
Citation: Mendoza EAV, Child JEM, Solano RR. Correlation of Erythrocyte Distribution Width \\
Values with Preeclampsia and Preeclampsia with Severity Criteria. Curr Inv Cln Med Res. \\
2022;2(1):1-4. DOI: 10.53902/CICMR.2022.02.000516
\end{tabular}


Currently, RDW has been studied as a cardiovascular biomarker, having its implication in diagnostic support of patients with heart failure, in a study Patel et al. showed that the increase in RDW is significantly associated with a decrease in erythrocyte deformity. It is plausible that a greater variation in the size of erythrocytes would increase blood viscosity, and concomitantly alter blood flow through the microcirculation, triggering or amplifying adverse consequences of a pre-existing vascular occlusion, in cardiovascular disease or venous thrombosis. Therefore, a strong association has been established between systemic arterial hypertension and red cell distribution width, however, few studies have been conducted comparing RDW in pregnant patients with results inconsistent, ${ }^{5,6}$ A study was carried out by Rojas, et al where the relationship of severe pre-eclampsia with platelet volume and RDW was documented, finding higher levels of RDW in patients with pre-eclampsia versus patients normotensive, ${ }^{7}$ on the other hand Raziye et al. I carry out a study finding levels higher RDWs in patients with severe pre-eclampsia, ${ }^{8}$ Reddy et al. I observe an increase in erythropoietin stimulation associated with placental hypoxia in patients with pre-eclampsia, the endothelial inflammatory process in pre-eclampsia leads to the destruction of erythrocytes and therefore hypoxia stimulating erythropoietin secretion. ${ }^{9}$ The red cell distribution width is an inexpensive and accessible marker; there are not enough studies that relate RDW to pre-eclampsia, so it is necessary to look for biochemical markers that support us with the prevention, diagnosis and prognosis of pre-eclampsia. The objective of this study was: to determine the correlation of the erythrocyte distribution width values with pre-eclampsia and pre-eclampsia with severity criteria.

\section{Materials and Methods}

A cross-sectional, descriptive, prospective and observational study was carried out. The population was made up of patients from the General Hospital "Dr. José María Rodríguez" who had the inclusion criteria: pregnancy equal to or greater than 20 weeks' gestation and diagnosis of preeclampsia without data on severity or preeclampsia with data on severity, a blood count, liver function tests, blood chemistry, as well as taking systolic and diastolic blood pressure upon admission to hospital. Exclusion criteria: patients with comorbidities, twin pregnancy. 39 patients were taken, representing the entire universe studied (30 patients with pre-eclampsia with data on severity and 9 patients with pre-eclampsia). An automated Coulter LH 780 Hematology Analyzer was used and the erythrocyte distribution width was calculated using the formula (standard deviation of MCV $\div$ mean corpuscular volume) x 100 . According to the literature found, normal RDW values between 11$15 \%$ are reported, therefore a RDW value greater than $15 \%$ was taken as abnormal. Statistical analysis was performed using the Student's t test to compare the RDW means of two groups and the
Spearman correlation to calculate the dependence between the non-parametric variables, the value of $\mathrm{p}<0.05$ was considered statistically significant.

\section{Results}

A total of 39 patients who met the inclusion criteria were studied, of which $9(23.07 \%)$ had pre-eclampsia without severity data and 30 (76.92\%) pre-eclampsia with severity data Table 1 . The most frequent severity data was by blood pressure figures (SAT $\geq 160 \mathrm{mmHg}$ and/or $\mathrm{TAD} \geq 110 \mathrm{mmHg}$ ), finding this severity data in 24 of the 30 patients who were classified as pre-eclampsia with severity data, that is, in $80 \%$. An increase in liver enzymes (TGP and/or TGP $\geq 70 \mathrm{U} / \mathrm{L}$ ) was found in the $23.33 \%$, symptoms (epigastric pain and / or severe maternal symptoms) in the $23.33 \%$, platelets $<100,000 \mathrm{xmcL}$ in $13.33 \%$ and creatinine $\geq 1.1 \mathrm{mg} / \mathrm{dl}$ in the $6.66 \%$ Table 2 . The mean red cell distribution width in patients with preeclampsia without data on severity was $14.86 \pm 0.92$ and $14.31 \pm 1.23$ in patients with preeclampsia with data on severity. Of the 9 patients with a diagnosis of pre-eclampsia without severity data, 5 had an increase in RDW levels, that is, in 55.55\%. In the case of the 30 patients diagnosed with pre-eclampsia with severity data, an increase in RDW was found in $33.33 \%$, that is, in 10 patients. When comparing the mean RDW levels of patients with pre-eclampsia without severity data (14.86 0.92) and the RDW of patients with pre-eclampsia with severity data $(14.31 \pm 1.23)$, we did not obtain a statistically significant difference $(\mathrm{p}=0.226)$. Therefore, we can infer that ADE is not related to the presence of pre-eclampsia with data on severity Table 3. Comparison of the mean RDW between patients with pre-eclampsia with severity data that presented an increase in systolic blood pressure as a severity criterion $(\geq 160 \mathrm{mmHg})$ and those that did not present it $(<160 \mathrm{mmHg})$. Of the 22 patients who presented systolic blood pressure figures as a criterion of severity, an increase in RDW levels was found in 10 patients (43.47\%). Of the 7 patients who did not present systolic blood pressure as a criterion of severity, none had an increase in RDW levels. Comparing the mean RDW in patients with systolic blood pressure as a criterion of severity (SAT $\geq 160 \mathrm{mmHg}$ ), with the mean RDW of the patients who did not present an increase in systolic blood pressure, we found an increase in RDW levels in the group with systolic blood pressure figures $\geq 160 \mathrm{mmHg}$ statistically significant ( $\mathrm{p}=0.042)$ Table 4.

A comparison was made of the mean RDW in patients with preeclampsia with severity data with decreased platelets $(<100,000)$ and patients with preeclampsia with severity data that did not present decreased platelets, 4 patients with preeclampsia with severity data presented plateletpenia, of these 4 patients, an increase in RDW was found in 2 patients (50\%). Of the 26 patients who did not present with platelet penia, 8 patients (30.76\%) presented increased levels of RDW. Regarding the liver profile, 7 patients 
with preeclampsia with data on severity presented an increase in GOT; of these 7 patients, 1 (14.28\%) presented increased levels of RDW. Of the 23 patients who did not present an increase in GOT, 9 (39.1\%) had an increase in RDW levels. Nevertheless, no statistically significant difference was found in the comparison of the mean RDW of the 2 groups. On the other hand, 7 patients presented an increase in TGP levels, of which one presented an increase in RDW (14.2\%). Of the 23 patients who did not present an increase in TGP, $9(39.13 \%)$ presented an increase in RDW. A higher mean RDW was found in the group of patients without a statistically significant increase in TGP ( $p=0.04)$. Elevation of creatinine levels was documented in 3 patients, of these 1 (33.33\%) presented an increase in RDW levels, 27 patients did not present changes in creatinine lev- els. Of the patients without changes in creatinine, 9 (33.33\%) suffered an increase in RDW levels. When comparing the mean RDW of both groups of patients, no significant difference was found. Simple linear regression was performed using the Spearman correlation since the studied variables presented a different distribution than the normal one, the above to corroborate the correlation between the levels of the red blood cell distribution width and systolic blood pressure figures, diastolic blood pressure figures, platelet, creatinine, transaminase, and protein levels. A weak positive correlation was found between red blood cell distribution width and systolic blood pressure, as well as with platelet and creatinine levels, and a weak negative correlation with diastolic blood pressure, TGP, TGP and protein levels.

Table 1: Selected patients Parameter.

\begin{tabular}{|l|l|l|}
\hline & $\begin{array}{l}\text { Preeclampsia without severity data } \\
\text { (n=9) }\end{array}$ & $\begin{array}{l}\text { Preeclampsia with severity data } \\
\text { (n=30) }\end{array}$ \\
\hline TAS (mmHg) & $145.5 \pm 7.2$ & $165.13 \pm 17.98$ \\
\hline TAD (mmHg) & $94.8 \pm 5.2$ & $106.26 \pm 10.58$ \\
\hline ADE (\%) & $14.86 \pm 0.92$ & $14.31 \pm 1.23$ \\
\hline platelets & $193.444 \pm 68.003$ & $185.5533 .33 \pm 72.971$ \\
\hline creatinine & $0.76 \pm 0.21$ & $0.75 \pm 0.22$ \\
\hline TGO & $30.2 \pm 17.1$ & $50.86 \pm 64.9$ \\
\hline TGP & $23.4 \pm 8.3$ & $57.3 \pm 82.82$ \\
\hline Dhl & $262.4 \pm 88.88$ & $246.1 \pm 103.46$ \\
\hline Urine protein (EGO) & $244.4 \pm 296.27$ & $128 \pm 198.16$ \\
\hline
\end{tabular}

Table 2: Frequency of patients who presented clinical or biochemical data of severity.

\begin{tabular}{|c|c|c|}
\hline Variable & Frequency & Percentage \\
\hline SAT $\geq 160 \mathrm{mmHg}$ & 22 & $73.33 \%$ \\
\hline $\mathrm{TAD} \geq 110 \mathrm{mmHg}$ & 17 & $56.66 \%$ \\
\hline Vasospasm facts & 4 & $13.33 \%$ \\
\hline Epigastralgia & 3 & $10 \%$ \\
\hline Creatinine $\geq 1.1 \mathrm{mg} / \mathrm{dl}$ & 2 & $6.66 \%$ \\
\hline $\mathrm{TGO}>70 \mathrm{~J} / \mathrm{L}$ & 7 & $23.33 \%$ \\
\hline $\mathrm{TGP}>70 \mathrm{U} / \mathrm{L}$ & 5 & $16.66 \%$ \\
\hline Platelets $<100,000 \mathrm{xmcL}$ & 4 & $13.33 \%$ \\
\hline
\end{tabular}

Table 3: Comparison of RDW between patients with pre-eclampsia without data on severity and patients with pre-eclampsia with data on severity.

\begin{tabular}{|c|c|c|}
\hline Patients with preeclampsia & Patients with preeclampsia with $\mathbf{P}$ severity data & $\mathbf{p = 0 . 2 2 6}$ \\
\hline Mean \pm SD & Mean $\pm S D$ & \\
\hline $14.86 \pm 0.92$ & $14.31 \pm 1.23$ & \\
\hline
\end{tabular}

Table 4: Comparison of RDW with systolic blood pressure Patients with $\mathrm{BP} \geq 160$ Patients with $\mathrm{BP}<160$.

\begin{tabular}{|c|c|c|}
\hline mmHg & mmHg & $\mathbf{P}=\mathbf{0 . 0 4 2}$ \\
\hline Mean \pm SD & Mean \pm SD & \\
\hline $14.59 \pm 1.17$ & $13.5 \pm 1.15$ & \\
\hline
\end{tabular}




\section{Discussion}

Elevated ADE levels are a reflection of a great heterogeneity in the size of erythrocytes, it is usually used to discriminate and differentiate between types of anemia, however, both excessive destruction and a deficit in the production of erythrocytes lead to an increase of its value. There are studies that show a close association between increased RDW and biomarkers of oxidative stress, inflammation, and kidney dysfunction. Proinflammatory and prothrombotic processes occur in preeclampsia, processes that can alter haematological parameters. ${ }^{10}$ Recently, ADE has been related to the presence and severity of hypertension. Patients with hypertension and prehypertension present levels elevated RDW, according to the literature, the highest RDW was found in patients with pre-eclampsia compared to normotensive patients, as well as higher RDW levels in patients with pre-eclampsia with severity data than in patients with preeclampsia. ${ }^{11}$ However, other studies such as that of Gezer, et al. They suggest that the role of RDW in early and late-onset preeclampsia has no significant implication as a marker. Inflammatory. ${ }^{12}$ In our study, when comparing patients with preeclampsia with severity data with systolic blood pressure values $\geq 160 \mathrm{mmHg}$ with patients with preeclampsia with severity data with blood pressure values less than $160 \mathrm{mmHg}$, we found a higher mean RDW in patients with blood pressure figures high systolic levels, which was statistically significant, the economic marker ADE that can be used as a prognosis, in our case the existing correlation of high blood pressure associated with a high ADE would denote as a criterion of severity, on the other hand, it opens a gap to continue the study of the association, since one of the limitations of this study is definitely the size of the sample, therefore, the findings found in the literature compared with the results of this study show a great discrepancy.

\section{Conclusion}

The identification of novel prognostic markers that are easily accessible and that do not imply an additional expense in their care and that can improve decision-making in patients with pre-eclampsia, in order to stratify the risk of these patients, in our study there is a relationship However, it encourages prospective studies to investigate its association and its implication with severity.

\section{Acknowledgement}

None

\section{Funding}

None.

\section{Conflicts of Interest}

The authors have no disclosures or conflicts of interest.

\section{References}

1. Sánchez E. Current status of preeclampsia in Mexico: from the epidemiological to its molecular mechanisms. Rev Invest Clin. 2010;62(3):252-260.

2. Laura A Magee, Anouk Pels, Michael Helewa, et al. Diagnosis, evaluation, and management of the hypertensive disorders of pregnancy. Pregnancy Hypertens. 2014;4(2):105-145.

3. González P, Guillermo Genaro Martínez Salazar, Omar García Nájera, et al. Preeclampsia, eclampsia, and HELLP. Rev Mex Anest. 2015;38 Suppl. S118-S127.

4. Salvagno G, Alessandra Picanza, Giuseppe Lippi, et al. Red blood cell distribution width: A simple parameter with multiple clinical applications. Crit Rev Clin Lab Sci. 2015;52(2):86-105.

5. Alcaino H, Mario Pavez, Humberto Toledo, et al. Red cell distribution width as a potential clinical biomarker in cardiovascular diseases. Rev Med Chile. 2016;144:634-642.

6. Patel K, Richard D Semba, Luigi Ferrucci, et al. Red cell distribution width and mortality in older adults: a meta-analysis. J Gerontol A Biol Sci Med Sci. 2010;65(3):258-265.

7. Jesús Antonio Viana Rojas, Alejandro Rosas Cabral, Jorge Prieto Macías, et al. Severity of preeclampsia and its relationship with platelet volume and red cell distribution width. Rev Med Inst Mex Seguro Soc. 2017;55(2):176-181.

8. Kurt R, et al. Relationship of red cell distribution width with the presence and severity of preeclampsia. Clin Appl Thromb Hemost. 2015;21(2):128-131.

9. Reddy S, et al. red cells distribution width was a marker of preeclampsia severity. J Evolution Med Dent Sci. 2016. 2016;5(48):3104-3107.

10. Shilpa Gopal Reddy, Harendra Kumar, Geetanjali Nagaraj, et al. Relationship between red cell distribution width (RDW) and short-term mortality in patients with acute coronary syndrome (ACS). Gac Med Mex. 2016;52:70-77.

11. Ahmed Bilal, Junaid H. Farooq, Immad Kiani, et al. Importance of mean red cell distribution width in hypertensive patients. Cureus. 8(11):e902.

12. Cenk Gezer, Atalay Ekin, Mehmet Özeren, et al. The role or the first trimester inflammation markers at early and late pre-eclampsia. Perinatal Journal. 2014;22(3):128-132. 